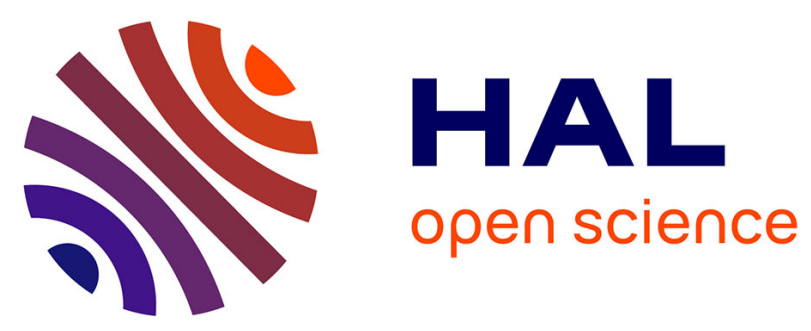

\title{
Validation of a new optimisation algorithm for registration tasks in medical imaging
}

Nicolas Wiest-Daesslé, Sylvain Prima, Christian Barillot

\section{To cite this version:}

Nicolas Wiest-Daesslé, Sylvain Prima, Christian Barillot. Validation of a new optimisation algorithm for registration tasks in medical imaging. IEEE International Symposium on Biomedical Imaging, Apr 2007, Washington, United States. 10.1109/ISBI.2007.356783 . inria-00133377

\section{HAL Id: inria-00133377 https://hal.inria.fr/inria-00133377}

Submitted on 26 Feb 2007

HAL is a multi-disciplinary open access archive for the deposit and dissemination of scientific research documents, whether they are published or not. The documents may come from teaching and research institutions in France or abroad, or from public or private research centers.
L'archive ouverte pluridisciplinaire HAL, est destinée au dépôt et à la diffusion de documents scientifiques de niveau recherche, publiés ou non, émanant des établissements d'enseignement et de recherche français ou étrangers, des laboratoires publics ou privés. 


\title{
VALIDATION OF A NEW OPTIMISATION ALGORITHM FOR REGISTRATION TASKS IN MEDICAL IMAGING
}

\author{
Nicolas Wiest-Daesslé $e^{1,2,3}$, Sylvain Prima ${ }^{1,2,3}$, Sean Patrick Morrissey ${ }^{1,2,3,4}$ and Christian Barillot ${ }^{1,2,3}$ \\ ${ }^{1}$ INRIA, VisAGeS U746 Unit/Project, IRISA, Campus de Beaulieu, F-35042 Rennes, France \\ ${ }^{2}$ University of Rennes I, CNRS IRISA, Campus de Beaulieu, F-35042 Rennes, France \\ ${ }^{3}$ INSERM, VisAGeS U746 Unit/Project, IRISA, Campus de Beaulieu, F-35042 Rennes, France \\ ${ }^{4}$ Department of Neurology, University hospital, Pontchaillou, rue Henri Le Guilloux, F-35033 Rennes, France
}

\begin{abstract}
A number of problems frequently encountered in brain image analysis can be conveniently solved within a registration framework, such as alignment of mono- or multi-sequence Magnetic Resonance Images (MRI) for single or multiple subjects, computation of the cerebral mid-sagittal plane in anatomical or diffusion-tensor MRI, correction of acquisition distortions in diffusion-weighted MRI, etc. A widely used approach for registration tasks consists of maximising a similarity criterion between the intensities of the images to be matched. In this context, efficient optimisation methods are needed to obtain good performances. In this paper, we introduce a new optimisation algorithm (called NEWUOA) to address the above registration problems, and we demonstrate its robustness and accuracy properties.
\end{abstract}

\section{INTRODUCTION}

For improved patients care, in many diseases, it is common to acquire longitudinal, multi-sequence MRI data for diagnosis, prognosis, or assessment of therapy. In this context, automated alignment of the MRI data is often required for a good assessment of tissue changes. A widely used approach for this issue is to maximise a global similarity criterion between the intensities of the images to be matched. Optimisation of such a criterion with respect to transformation parameters in a fast, robust and accurate manner is then critical. In a previous paper [1], we proposed and validated a new optimisation algorithm for rigid-body multi-modal image registration. This New Unconstrained Optimisation Algorithm (NEWUOA) was initially introduced by M.J.D. Powell [2] as a fast method to deal with high dimensional problems. We showed [1] that NEWUOA is much faster, but also more robust and more accurate than two other derivative-free algorithms such as the Nelder-Mead's downhill simplex (SMP) and the Powell's direction set method (POW) as implemented in Press et al. [3].

Here, in this paper, we investigate NEWUOA as a general optimisation tool for different problems frequently encoun- tered in medical image processing, such as: 1) rigid and 2) linear normalisation of single or multiple subjects in a common reference frame; 3) computation of the cerebral midsagittal plane (MSP) for a) greyscale images and b) diffusion tensor MRI (DT-MRI) and 4) correction of distortions in diffusion-weighted MRI (DW-MRI). In Section 2.1, we expose a general formulation for the four above mentioned registration procedures. In Section 2.2, we distinctly describe the parameterisation of the transformations involved in each of these procedures. We give a brief overview of NEWUOA in Section 2.3. We validate its accuracy and robustness in Section 3 , both quantitatively and qualitatively, on synthetic and real data. We discuss our results and conclude in Section 4.

\section{METHODS}

\subsection{A General formulation for four registration problems}

Registration procedures can be addressed in an optimisation framework, in which the optimal transformation $\widehat{T}$ is defined as:

$$
\widehat{T}=\underset{T}{\arg \min } \Delta(I, T(J))
$$

where $I$ is a reference image, $J$ is a floating image, $T(J)$ is the image $J$ transformed using the transformation $T$, and $\Delta$ is a global similarity measure between the intensities of $I$ and $T(J)$ on their overlapping area.

In this article we focus on four particular registration types:

1) rigid registration :

- $T=R$ : rigid-body transformation,

- $I$ and $J$ : two images of the same patient either at different time points or from different modalities,

2) linear registration:

- $T=A$ : linear transformation,

- $I$ and $J$ : two images of different patients,

3) mid-sagittal plane computation:

- $T=S$ : reflection symmetry with respect to a plane, 
- $I=J: I$ is either a) a greyscale image or b) a DT-MRI image.

4) distortion correction for DW-MRI:

- $T=D: \mathrm{D}$ is a slice-dependent piecewise affine transformation,

- $I$ : diffusion-unweighted image and $J$ : diffusionweighted image.

The procedures 1, 2, 3.a and 4 involve greyscale images. In these cases the similarity criterion is mutual information. The problem 3.b involves DT-MRI images. In this case the similarity measure is simply the mean Frobenius distance between the tensors. Experimentally, this measure has the same accuracy and robustness as other measures theoretically better suited for tensor data (log-Euclidean or geodesic distances) but is much faster to compute.

\subsection{Parameterisation of the transformations}

\subsection{1. rigid registration}

A rigid transformation $R$ moves each point $p$ to $p^{\prime}$ following:

$$
p^{\prime}=R(p)=\mathbf{R}_{\mathbf{x}}\left(\phi_{x}\right) \mathbf{R}_{\mathbf{y}}\left(\phi_{y}\right) \mathbf{R}_{\mathbf{z}}\left(\phi_{z}\right) p+\mathbf{t},
$$

where $\mathbf{R}_{\mathbf{x}}$ (resp. $\mathbf{R}_{\mathbf{y}}$ and $\mathbf{R}_{\mathbf{z}}$ ) is a rotation around the $x-$ (resp. $y-$ and $z-$ ) axis and $\mathbf{t}=\left(t_{x}, t_{y}, t_{z}\right)$ is a translation.

\subsection{2. linear registration}

A linear transformation can be expressed as:

$$
p^{\prime}=A(p)=\mathbf{R}_{\mathbf{x}}\left(\phi_{x}\right) \mathbf{R}_{\mathbf{y}}\left(\phi_{y}\right) \mathbf{R}_{\mathbf{z}}\left(\phi_{z}\right) \mathbf{G S} p+\mathbf{t}
$$

where $\mathbf{G}$ and $\mathbf{S}$ are shear and scale matrices.

\subsection{3. mid-sagittal plane computation}

The computation of the mid-sagittal plane, $P$, can be implemented as a registration scheme [4] if we consider $P$ as the plane that maximises the similarity of the image by reflective symmetry. $P$ is parameterised by three variables: its distance $d$ to the origin and a normal unit vector $\mathbf{n}$, characterised by two angles $\alpha$ and $\beta$ :

$$
\mathbf{n}^{t}=\left[\begin{array}{lll}
\cos (\beta) \cos (\alpha) & \sin (\beta) \cos (\alpha) & \sin (\alpha)
\end{array}\right]
$$

Let $p^{\prime}$ be the reflection of $p$ with respect to the plane $P$, that is $p^{\prime}=S(p)$ where $S$ is the reflection symmetry with respect to $P$. It can be shown that:

$$
p^{\prime}=S(p)=\mathbf{H} p+\mathbf{t}=\left(\mathbf{I}-2 \mathbf{n n}^{t}\right) p+2 d \mathbf{n}
$$

\subsection{4. correction of distortions for diffusion-weighted image}

DW-MR images will be misaligned when acquired with distortions artifacts. Therefore, correction of these distortions is of great importance for the estimation of a precise tensor at each point of the image grid. We use the modelisation of the distortion proposed by Netsch [5], which is the combination of a rigid transformation and a slice-dependent scale, shear and translation transformation. The transformation for the correction of distortions is given by:

$$
p^{\prime}=D(p)=R(M(p)),
$$

where $M(p)=\mathbf{R}_{\psi} p+\mathbf{t}_{\psi}$. In the case of axial slices, the $\mathbf{R}_{\psi}$ matrix and $\mathbf{t}_{\psi}$ vector are given by:

$$
\mathbf{R}_{\psi}=\left[\begin{array}{ccc}
1 & 0 & 0 \\
\alpha & \beta & 0 \\
0 & 0 & 1
\end{array}\right], \mathbf{t}_{\psi}=\left[\begin{array}{l}
0 \\
\gamma \\
0
\end{array}\right]
$$

with $\alpha=\psi_{1} z^{2}+\psi_{2} z+\psi_{3}, \beta=\psi_{4} z^{2}+\psi_{5} z+\psi_{6}+1$ and $\gamma=\psi_{7} z^{2}+\psi_{8} z, z$ being the slice coordinate.

As a summary, we have:

1. 6 parameters for the rigid registration: $\left(\phi_{x}, \phi_{y}, \phi_{z}, t_{x}, t_{y}, t_{z}\right)$

2. 12 parameters for the linear registration: $\left(\phi_{x}, \phi_{y}, \phi_{z}, t_{x}, t_{y}, t_{z}, s_{x}, s_{y}, s_{z}, g_{x}, g_{y}, g_{z}\right)$

3. 3 parameters for the mid sagittal plane estimation: $(\alpha, \beta, d)$

4. 14 parameters for the correction of distortions: $\left(\phi_{x}, \phi_{y}, \phi_{z}, t_{x}, t_{y}, t_{z}, \psi_{1}, \psi_{2}, \psi_{3}, \psi_{4}, \psi_{5}, \psi_{6}, \psi_{7}, \psi_{8}\right)$

\subsection{NEWUOA}

The New Unconstrained Optimisation Algorithm (NEWUOA) is an optimisation algorithm recently proposed by Powell [2]. Here we briefly outline the method:

- Create a quadratic model $Q$ of the function $F$ to optimise within a trust-region; $Q$ interpolates $F$ at some chosen sample points,

- Do the following iterations:

- Compute the minimum of $Q$ within the trust-region,

- Update the model using the minimum,

- Stop if the latest trust-region radius is lower than the user-defined end value,

- Stop if the distance between $Q$ and $F$ is small enough (perfect match of the model and the objective function $F$ ),

- Decrease the trust-region radius, if the values computed for $F$ stops decreasing.

The initial radius of the trust region is set to $5 \mathrm{~mm}$ (or degree) for each problem. The algorithm iterates until a minimum trust-region radius is reached $\left(10^{-3} \mathrm{~mm}\right.$ (or degree) in our experiments). 


\section{VALIDATION}

The validation of procedures 1,2 and 3 is performed with a ground truth transformation for perfectly aligned images. In our experiments, we generated random ground truth transformation: 50 for the rigid registration and 100 for the other problems. We defined two measures to quantify: 1) the capture range of the algorithm (i.e. the distance between $T$ and the identity transformation) and 2) its accuracy (i.e. the distance between $T$ and $\widehat{T}$ ). The robustness is evaluated by computing the number of results giving accuracy lower than the image resolution. Finally for the correction of distortions in DW-MRI (problem 4), we visually assessed the quality of the correction with real images.

\section{1. rigid registration}

In this section, the images are the BrainWeb [6] T1- and T2weighted MR images. These phantom images are corrupted with Rician noise $(9 \%)$. The spatial resolution for each volume is $1 \times 1 \times 1 \mathrm{~mm}^{3}$ and the size of each volume is $181 \times$ $217 \times 181$ voxels. For our validation, we used the scheme proposed by Thevenaz et al. [7]. We defined a measure of the transformation error, the warping index, defined as follows: $J$ and $I$ are the BrainWeb T1- and T2-weighted MR images in perfect alignment. We computed two transformed images $I_{T}$ and $J_{T}$, as following: $I_{T}(x)=I(T(x))$ and $J_{T}(x)=$ $J\left(T^{-1}(x)\right)$, where $T$ is a random transformation. The two transformations introduce similar interpolation artifacts in both images. The registration process shall recover $R=T \circ T$ which aligns $J_{T}$ with $I_{T}$. Let $\widehat{R}$ be the estimated transformation.

The warping index $\omega 1$ measures the average geometric error between $R$ and $\widehat{R}$ :

$$
\omega 1=\frac{1}{\# \Omega\left(I_{T}\right)} \sum_{x \in \Omega\left(I_{T}\right)}\left\|R^{-1}(x)-\widehat{R}^{-1}(x)\right\|,
$$

$\Omega\left(I_{T}\right)$ being the image grid of $I_{T}$ and $\|-\|$ the Euclidean norm. A measure of the initial distance between the images to register is computed using the measure modified as follow:

$$
\delta 1=\frac{1}{\# \Omega\left(I_{T}\right)} \sum_{x \in \Omega\left(I_{T}\right)}\left\|R^{-1}(x)-x\right\|
$$

\section{2. linear registration}

The validation of linear registration is the same as for rigid registration. However, the warping index $\omega 1$ is not well suited for linear registration because it depends on the image $I_{T}$ and thus on $T$. For two different initial random linear transformations $T_{1}$ and $T_{2}$ (with different shears and scales) the warping indices computed over $I_{T_{1}}$ and $I_{T_{2}}$ will not have the same magnitude, and thus cannot be compared to each other. We introduced a new warping index, defined with respect to $I$ :

$$
\omega 2=\frac{1}{\# \Omega(I)} \sum_{x \in \Omega(I)}\left\|T \circ \widehat{R}^{-1} \circ T(x)-x\right\|,
$$

$\Omega(I)$ being the image grid of $I$ and $\|-\|$ the Euclidean norm. This new warping index allows the measure of the error in a scaling and shearing independent manner. We defined the initial distance as:

$$
\delta 2=\frac{1}{\# \Omega(I)} \sum_{x \in \Omega(I)}\|T \circ I d \circ T(x)-x\|,
$$

with $I d$ the identity transformation.

\begin{tabular}{|c|c|c|c|}
\hline & dist. $(\mathrm{mm})$ & acc. $(\mathrm{mm})$ & rob. \\
\hline Rigid & $\delta 1=27 \pm 10$ & $\omega 1=0.1 \pm 0.05$ & $49 / 50$ \\
\hline Linear & $\delta 2=35 \pm 13$ & $\omega 2=0.2 \pm 0.06$ & $100 / 100$ \\
\hline MSP (MRI) & $\delta 3=40 \pm 14$ & $\omega 3=0.11 \pm 0.1$ & $73 / 100$ \\
\hline MSP (DT-MRI) & $\delta 3=40 \pm 14$ & $\omega 3=0.08 \pm 0.08$ & $96 / 100$ \\
\hline
\end{tabular}

Table 1. Capture range, accuracy and robustness of the algorithm.

\section{3. mid-sagittal plane computation (MSP)}

In this section, either a BrainWeb (problem a.) or a real DTMR image (problem b.) was used. In these images the MSP of the brain was already well aligned with the centre of the image grid. Half of the image was removed and replaced by the flipped version of the other half. In this new image, the center of the grid was a perfect symmetry plane. A random rigid transformation was applied to the image. The accuracy measures $\delta 3$ and $\omega 3$ were used to evaluate the capture range (i.e. the maximal tilt that it is able to correct) and the accuracy (i.e. the closeness between the estimated plane and the ground truth plane) of the algorithm (see Prima et al. [8] for details).

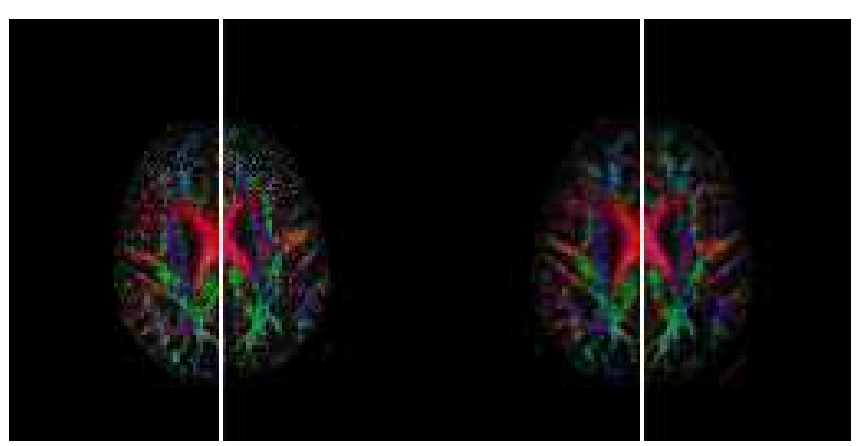

Fig. 1. Computation of the mid-sagittal plane in DT-MRI in a patient with Parkinson's disease (left: original image and right: same image with the computed plane aligned with the image mid-plane). The color coding is: red for left-right, green for antero-posterior and blue for top-bottom. It is applied to the principal diffusion of direction (eigenvector of the diffusion tensor with the largest eigenvalue), with the brightness proportional to the fractional anisotropy (FA). 


\section{4. correction of distortion for diffusion-weighted image}

Since no DW-MRI phantom is available, we used a real dataset, and visual interpretation of the results to estimate the quality of the correction of distortions for DW-MRI. Figure 2 shows the computed FA maps for the original image and for the distortion corrected images. The arrows in the zoomed-in region show areas where the effects of the correction of distortions are the most visible.

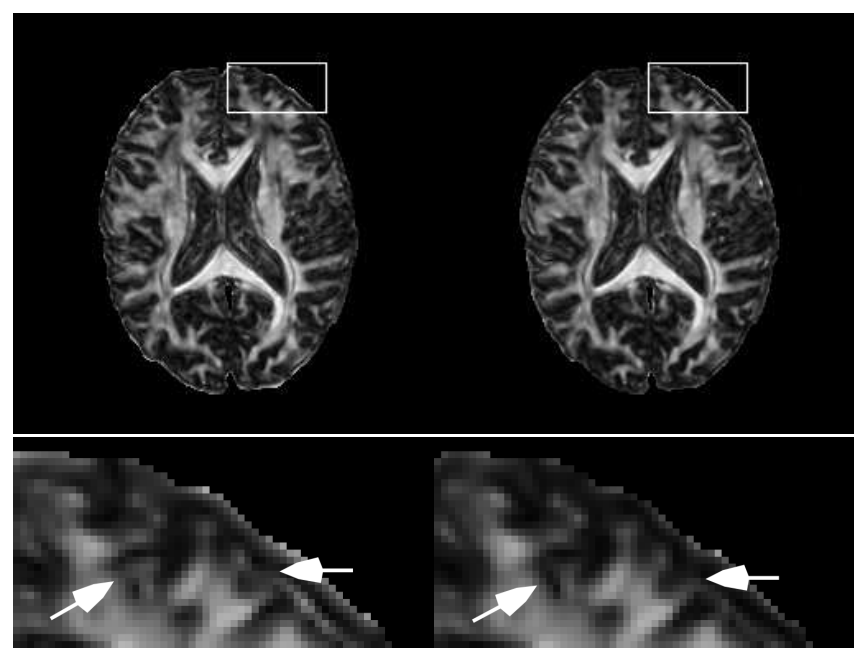

Fig. 2. Fractional anisotropy maps. Top left: original image. Top right: distortion corrected image. The correction of distortion artifacts results in sharper contrasts for FA maps, mostly in sulcal regions. Bottom: Zoom-in on the fractional anisotropy maps. Bottom left: original image. Bottom right: distortion corrected image. The edges are enhanced if the DWI image is well aligned (see arrows).

\section{DISCUSSION}

We introduced an optimisation algorithm, NEWUOA, in the context of registration procedures for brain images. NEWUOA was validated on a synthetic data set and checked with routine images. We focused on its accuracy and robustness properties addressing four different registration tasks (rigid, linear, midsagittal plane computation and piecewise linear registration). The capture range measured (with mean between 27 and 40 $\mathrm{mm}$ for our experiments) is high enough to deal with routine data. In a previous work [1] we compared the computation time of NEWUOA for rigid registration with two widely used algorithms (Nelder-Mead's downhill simplex (SMP), Powell's direction set method (POW)). In this paper NEWUOA performed much faster than the SMP and POW. Testing for the other three problems, NEWUOA was also faster than the other two algorithms. The study of the automated computation of the mid-sagittal plane of the brain was conducted with greyscale and DT-MRI images. Using a synthetic dataset, we demonstrated that it is both robust and accurate. The realign- ment of this plane improves the display of DT-MRI data. In terms of accuracy, the computation of the plane in DT-MRI has a slightly better mean, but a comparison between the DTMRI mid-sagittal plane and the plane defined on the corresponding anatomical images needs to be performed. The midsagittal plane computation might provide useful constraints for a registration or segmentation process. The correction of distortions for DW-MR image is required for a good estimation of the tensor parameters, since misalignment has an important impact on study results of brain structures using tensor information. Tensor coefficients undergo significant changes if the images are misaligned, thus biasing the results. The robustness and accuracy of NEWUOA was not tested for the correction of distortions, but the qualitative inspection of real data demonstrated enhanced edges on different diffusion maps extracted from the datasets. Preliminary results showed that the correction of the distortions using other algorithms (POW and SMP) resulted in transformations close to rigid-body transformations, which are unlikely to correct any distortion. On the contrary, the transformations found by NEWUOA are truly piecewise linear. To sum up, our results corroborate that NEWUOA is an accurate and robust algorithm for different kinds of registration problems.

\section{REFERENCES}

[1] N. Wiest-Daesslé et al., "Evaluation of a new optimisation algorithm for rigid registration of MRI data," in SPIE Medical Imaging 2007: Image Processing, 2007, to be published.

[2] M.J.D. Powell, "The NEWUOA Software for Unconstrained Optimization without Derivatives," in Workshop On Large Scale Nonlinear Optimization, 2004, vol. 83 of Nonconvex Optimization and Its Applications.

[3] William H. Press et al., Numerical Recipes: The Art of Scientific Computing, Cambridge University Press, 1992.

[4] S. Prima and N. Wiest-Daesslé, "Computation of the MidSagittal Plane in Diffusion Tensor MR Brain Images," in SPIE Medical Imaging 2007: Image Processing, 2007, to be published.

[5] T. Netsch and A. van Muiswinkel, "Quantitative evaluation of image-based distortion correction in diffusion tensor imaging.," IEEE Trans. Med. Imaging, vol. 23, no. 7, pp. 789-98, 2004.

[6] D. L. Collins et al., "Design and construction of a realistic digital brain phantom.," IEEE Trans. Med. Imaging, vol. 17, no. 3, pp. 463-468, 1998.

[7] P. Thévenaz and M. Unser, "Optimization of mutual information for multiresolution image registration," IEEE Trans. on Image Processing, vol. 9, no. 12, pp. 2083-2099, Dec. 2000.

[8] S. Prima et al., "Computation of the mid-sagittal plane in 3D brain images," IEEE Trans. Med. Imaging, vol. 21, no. 2, pp. 122-138, 2002. 